\title{
Transaortic mitral valve secondary chordal cutting in patients with obstructive hypertrophic cardiomyopathy and mild septal hypertrophy
}

\author{
Irene Binaco, Paolo Spirito, Daniele Poggio, Valter Casati, Massimiliano Grillo, Paolo Ferrazzi \\ Hypertrophic Cardiomyopathy Center, Policlinico di Monza, Monza, Italy \\ Correspondence to: Paolo Ferrazzi, MD. Centro per la Cardiomiopatia Ipertrofica e le Cardiopatie Valvolari, Policlinico di Monza, Via Amati 111, \\ Monza 20090, Italy. Email: paolo.ferrazzi@policlinicodimonza.it.
}

Submitted Feb 08, 2017. Accepted for publication Apr 04, 2017.

doi: 10.21037/acs.2017.04.05

View this article at: http://dx.doi.org/10.21037/acs.2017.04.05

\section{Clinical vignette}

A proportion of patients with obstructive hypertrophic cardiomyopathy (HCM) and severe heart failure symptoms have only mild septal hypertrophy (1). In such patients, mitral valve (MV) abnormalities play an important role in systolic MV leaflets displacement into the left ventricular LV outflow tract and blood flow obstruction (1-3). Therefore, conventional septal myectomy alone may not be sufficient to relieve LV obstruction and symptoms, and often $M V$ repair or replacement is the surgical alternative (1). Transaortic cutting of MV secondary chordae is a novel technique for MV repair that, associated with a shallow septal myectomy, abolishes the outflow gradient, relieves heart failure symptoms, and avoids MV replacement in patients with obstructive HCM and mild septal thickness (4).

A 45-year-old female patient with obstructive HCM, mild septal hypertrophy and severe heart failure symptoms (New York Heart Association functional class III) unresponsive to medications was referred to our institution for surgical treatment of LV outflow obstruction. Physical examination revealed blood pressure $124 / 72 \mathrm{mmHg}$ and heart rate of $72 \mathrm{BPM}$ on bisoprolol $10 \mathrm{mg} /$ day. Transthoracic echocardiography showed mild septal hypertrophy $(17 \mathrm{~mm})$ confined mainly to the basal and medium portion of the anterior septum, with marked systolic anterior motion of the MV leaflets and leaflet-septal contact at rest, and moderate left atrial dilatation. Doppler echocardiography showed an LV outflow maximal gradient of $72 \mathrm{mmHg}$ under basal conditions, moderate-to-severe MV regurgitation, and a systolic pulmonary pressure of $32 \mathrm{mmHg}$. Cardiac magnetic resonance documented a small area of intramural delayed enhancement on the anterior septum. Coronary angiography was normal. Because of severe outflow gradient and heart failure symptoms, relatively mild septal thickness, MV leaflets with anterior leaflet tenting, and important MV regurgitation, we planned a shallow surgical myectomy, possibly associated with MV secondary chordal cutting through a median sternotomy.

\section{Surgical technique}

\section{Preparation}

After induction of general anaesthesia, intra-operative transesophageal echocardiography (TEE) is performed to assess magnitude and distribution of septal hypertrophy, and carefully inspect the MV features and morphology and function of the MV apparatus. The heart is then arrested and protected by infusion of anterograde warm blood cardioplegia into the aortic root. During the first dose of cardioplegia, $1 \mathrm{mg} / \mathrm{kg}$ of esmolol is injected into the reservoir of the extracorporeal circulation system. Additional doses of cardioplegia are administered into the right and left coronary ostia every 20 minutes. Starting from the second cardioplegia, the dosage of esmolol is decreased to $0.5 \mathrm{mg} / \mathrm{kg}$.

\section{Operation}

Septal myectomy is performed through an aortotomy, 
as previously reported (5). In particular, to allow better exposure of the ventricular septum during myectomy, a double transverse stitch is positioned in correspondence of the area of most marked septal thickness, as assessed with pre-operative transthoracic echocardiography and intraoperative TEE. This area is usually located at the level of the fibrotic contact plaque. Great care is taken to ensure that the length (from base to apex) of the incision goes beyond the point of mitral-septal contact (Video 1). The septal myocardial tissue is excised in a single piece in order to leave a smooth surface on the remaining septum (Video 1) (5). Fibrous or abnormal structures connecting the papillary muscles to the ventricular septum or LV free wall are systematically excised and/or dissected.

Subsequently, a valve analysis of the anterior mitral leaflet ventricular surface is performed. Using two forceps, the anterior leaflet is pushed towards the left atrium to identify retracted secondary chordae and lines of traction exercised by these chordae on the leaflet. The abnormal traction of secondary chordae on papillary muscle is usually confirmed by presence of fibrosis at the chordal attachment to the papillary muscle. Fibrotic secondary chordae are then selected with a nerve hook. The corresponding primary chordae (located in front of the secondary chordae) are also identified to confirm that primary chordae support the anterior leaflet free margin. The distal extreme of the selected fibrotic secondary chordae is then cut from its connection to the papillary muscle and the proximal extreme is cut from the anterior leaflet (Video 1). In the present case, 3 fibrotic secondary chordae considered important in tethering the anterior leaflet towards the outflow tract were cut.

\section{Completion}

The incision on the ascending aorta is closed, aorta declamped, and cardiac rhythm restored. TEE is repeated off cardiopulmonary bypass to detect a residual outflow tract gradient and aortic or MV regurgitation, and to exclude surgical complications such as ventricular septum perforation. In the present case, TEE shows that the MV coaptation point has moved away from the LV outflow tract to a more posterior and normal position within the $\mathrm{LV}$ cavity, MV systolic anterior motion is absent and residual MV regurgitation trivial (Video 1). The sternotomy is closed in a standard fashion.

\section{Comment}

We have previously demonstrated that transaortic cutting of thickened MV secondary chordae, in association with a shallow myectomy, moves the MV coaptation point away from the LV outflow tract to a more posterior position within the LV cavity by abolishing anterior leaflet tethering and restoring slack primary chordae to their function (4). These changes in MV motion increase the outflow tract size, prevent $\mathrm{MV}$ displacement into the ejection blood flow and contribute to abolition of outflow obstruction. Our subsequent experience in more than 100 patients who have undergone cutting of thickened MV secondary chordae, in association with septal myectomy, has confirmed the favorable results reported in our initial study (4), with a hospital mortality $<1 \%$, abolition or substantial decrease in $\mathrm{LV}$ outflow gradient (to $<30 \mathrm{mmHg}$ ), relief of heart failure symptoms in $>90 \%$ of patients, and substantial decrease of pre-operative MV regurgitation. MV flail or prolapse has never been documented at most recent evaluation.

A caveat of secondary chordal cutting is the addition of about 15 minutes to the aortic cross-clamping time of standard septal myectomy. Selection of the secondary chordae responsible for tethering the anterior leaflet also requires expertise. Previous experience with $M V$ repair may contribute to reduce the learning curve for chordal cutting.

\section{Acknowledgements}

None.

\section{Footnote}

Conflicts of Interest: The authors have no conflicts of interest to declare.

\section{References}

1. Gersh BJ, Maron BJ, Bonow RO, et al. 2011 ACCF/AHA guideline for the diagnosis and treatment of hypertrophic cardiomyopathy: executive summary: a report of the American College of Cardiology Foundation/American Heart Association Task Force on Practice Guidelines. Circulation 2011;124:2761-96.

2. Dearani JA, Ommen SR, Gersh BJ, et al. Surgery insight: Septal myectomy for obstructive hypertrophic cardiomyopathy--the Mayo Clinic experience. Nat Clin Pract Cardiovasc Med 2007;4:503-12. 
3. Desai MY, Bhonsale A, Smedira NG, et al. Predictors of long-term outcomes in symptomatic hypertrophic obstructive cardiomyopathy patients undergoing surgical relief of left ventricular outflow tract obstruction. Circulation 2013;128:209-16.

4. Ferrazzi P, Spirito P, Iacovoni A, et al. Transaortic Chordal
Cutting: Mitral Valve Repair for Obstructive Hypertrophic Cardiomyopathy With Mild Septal Hypertrophy. J Am Coll Cardiol 2015;66:1687-96.

5. Iacovoni A, Spirito P, Simon C, et al. A contemporary European experience with surgical septal myectomy in hypertrophic cardiomyopathy. Eur Heart J 2012;33:2080-7.

Cite this article as: Binaco I, Spirito P, Poggio D, Casati V, Grillo M, Ferrazzi P. Transaortic mitral valve secondary chordal cutting in patients with obstructive hypertrophic cardiomyopathy and mild septal hypertrophy. Ann Cardiothorac Surg 2017;6(4):426-428. doi: 10.21037/acs.2017.04.05 